J Prosthodont Res. 2016 April ; 60(2): 131-137. doi:10.1016/j.jpor.2015.12.006.

\title{
Effect of a dietary supplement on peri-implant bone strength in a rat model of osteoporosis
}

\author{
Takahiro Takahashi, DDS ${ }^{1}$, Takehiro Watanabe, DDS, PhD², Hiroshi Nakada, DDS, $\mathrm{PhD}^{2}$, \\ Yasuhiro Tanimoto, $\mathrm{PhD}^{3}$, Suguru Kimoto, DDS, $\mathrm{Phd}^{2}$, Dindo Q. Mijares, $\mathrm{MS}^{4}$, Yu Zhang, \\ $\mathrm{PhD}^{4}$, and Yasuhiko Kawai, DDS, MMedSci, PhD $^{2}$ \\ ${ }^{1}$ Nihon University Graduate School of Dentistry at Matsudo, Removable Prosthodontics, Matsudo, \\ Japan
}

${ }^{2}$ Department of Removable Prosthodontics, Nihon University of Dentistry at Matsudo, Japan

${ }^{3}$ Department of Dental Biomaterials, Nihon University of Dentistry at Matsudo, Japan

${ }^{4}$ Department of Biomaterials \& Biomimetics, New York University College of Dentistry, U.S.A

\begin{abstract}
Purpose-Osteoporosis contributes to impaired bone regeneration and remodeling through an imbalance of osteoblastic and osteoclastic activity, and can delay peri-implant bone formation after dental implant surgery, resulting in a prolonged treatment period. It poses several difficulties for individuals with large edentulous areas, and decreases their quality of life. Consequently, prompt postoperative placement of the final prosthesis is very important clinically. Peri-implant bone formation may be enhanced by systemic approaches, such as the use of osteoporosis supplements, to promote bone metabolism. We aimed to confirm whether intake of synthetic bone mineral (SBM), a supplement developed for osteoporosis, could effectively accelerate peri-implant bone formation in a rat model of osteoporosis.

Methods-Thirty-six 7-week-old ovariectomized female Wistar rats were randomly assigned to receive a standardized diet with or without SBM (Diet with SBM group and Diet without SBM group, respectively; $\mathrm{n}=18$ for both). The rats underwent implant surgery at 9 weeks of age under general anesthesia. The main outcome measures, bone mineral density (BMD) and pull-out strength of the implant from the femur, were compared at 2 and 4 weeks after implantation using the Mann-Whitney U test.
\end{abstract}

Results-Pull-out strength and BMD in the Diet with SBM group were significantly greater than those in the Diet without SBM group at 2 and 4 weeks after implantation.

Corresponding author: Dr. T. Watanabe, Department of Removable Prosthodontics, Nihon University School of Dentistry, at Matsudo, Matsudo, 2-870-1 Sakaecho-nishi, Chiba 271-8587, Japan, takehiro.watanabe1018@gmail.com.

Conflict of interest statement

No conflict of interest exists.

Publisher's Disclaimer: This is a PDF file of an unedited manuscript that has been accepted for publication. As a service to our customers we are providing this early version of the manuscript. The manuscript will undergo copyediting, typesetting, and review of the resulting proof before it is published in its final citable form. Please note that during the production process errors may be discovered which could affect the content, and all legal disclaimers that apply to the journal pertain. 
Conclusions-This study demonstrated that SBM could be effective in accelerating peri-implant bone formation in osteoporosis.

\section{Keywords}

Animal study; Implant; Healing period; Supplement; Bone formation

\section{Introduction}

Japan is known for having the world's most rapidly aging population; individuals above the age of 65 years account for $25 \%$ of the total population, and number over 30 million [1]. The trend toward an increasingly elderly population is evident not only in Japan and other developed countries, but also in nations across the globe, attesting to ongoing improvements in health, welfare, diet, and other factors [2]. In an aging society, even elderly individuals are targeted for dental implant treatment to improve quality of life.

In one investigation, $80 \%$ of patients who received implant treatment reported that the treatment period was too long [3], suggesting that shortening of the treatment period is an important factor in improving this therapy. However, osseointegration between the implant and bone requires at least 3-6 months [4]. Furthermore, the elderly are at risk of osteoporosis, a condition characterized by a severe decrease in bone mineral density (BMD) that results in reduced bone strength and alterations in trabecular bone characteristics [5]. Osteoporosis also contributes to impaired bone regeneration and remodeling through an imbalance in osteoblastic and osteoclastic activity, and may delay peri-implant bone formation [6,7]. It poses several difficulties for individuals with large edentulous areas, and decreases their quality of life. Thus, it is very important to study approaches for accelerating bone formation around dental implants in patients with osteoporosis.

Several strategies to accelerate bone formation after implant placement have been reported, the simplest of which is immediate implant restoration with immediate implant placement $[8,9]$. However, few patients undergo this treatment, as only those with adequate bone strength and bone volume are candidates. Other approaches have involved improving the interface between the implant and the peri-implant bone tissue. Implant materials and/or implant surface treatments have been developed to augment cell adhesion and protein adsorption at the implant-bone tissue interface [10,11]. Ogawa [12] reported that titanium surfaces treated with ultraviolet light developed a unique electrostatic status and acted as direct cell attractants to effectively reduce the osseointegration period without the aid of ionic or organic bridges, which is a novel physicochemical characteristic of titanium [13]. Vibratory stimulation has also been reported to improve the bone-to-implant contact ratio [14].

In addition to these local approaches to improve osseointegration between the implant body and surrounding bone tissue, systemic approaches such as the use of osteoporosis medication to promote bone metabolism may improve peri-implant bone formation $[15,16]$. LeGeros developed synthetic bone mineral (SBM), a calcium-phosphate-based supplement incorporating magnesium $(\mathrm{Mg})$, zinc $(\mathrm{Zn})$, fluoride $(\mathrm{F})$, and carbonate, to promote bone formation and inhibit bone resorption in osteoporosis [15]. On the basis of this background, 
we previously conducted studies revealing that SBM accelerates bone formation in normal rats both with and without implant placement [17][15].

As a next step in this research, the current study was conducted to assess whether SBM could effectively accelerate peri-implant bone formation in a rat model of osteoporosis. The null hypothesis was that bone formation evaluated by BMD and pull-out strength would not significantly differ between osteoporosis rats fed diets with and without an SBM supplement.

\section{Materials and methods}

\subsection{Animal diet}

AIN-93M, developed by the American Institute of Nutrition Committee and prepared by Oriental Yeast Co., Ltd. (Tokyo, Japan), was used as the control diet. The experimental diet consisted of AIN-93M and SBM. SBM was prepared according to LeGeros' protocol [18]. Briefly, a mixture of dicalcium phosphate dihydrate $\left(\mathrm{CaHPO}_{4} \cdot 2 \mathrm{H}_{2} \mathrm{O}\right)$ and magnesium and zinc chlorides $\left(\mathrm{MgCl}_{2}\right.$ and $\mathrm{ZnCl}_{2}$, respectively) were hydrolyzed in double-distilled water containing dissolved potassium carbonate and sodium fluoride. Thereafter, SBM was added to AIN-93M, the mineral composition of which was adjusted using Mijares' method [19]. The compositions of the diets with and without SBM are shown in Table 1.

\subsection{Animal experiments}

The study protocol was approved by the Ethical Committee of Nihon University (AP14MD018). Thirty-six 6-week-old female ovariectomized (OVX) Wistar rats (Sankyo Labo Service, Tokyo, Japan) were included. Seven-week-old rats were fed a diet without SBM for 1 week to acclimate them to environmental changes, then randomly allocated into one of two groups: a control group fed a diet without SBM (Diet without SBM group, $\mathrm{n}=18$ ) or an experimental group fed a diet with SBM (Diet with SBM group, $\mathrm{n}=18$ ). Rats were housed individually; food and water were given ad libitum, and temperature and relative humidity were maintained at $20^{\circ} \mathrm{C} \pm 1^{\circ} \mathrm{C}$ and $50 \% \pm 1 \%$, respectively.

All rats underwent implant surgery on their femurs at 9 weeks old while under general anesthesia administered via an intraperitoneal injection of medetomidine, midazolam and butorphanol. One operator prepared a hole in the femur $1.2 \mathrm{~mm}$ in diameter and $2.5 \mathrm{~mm}$ deep using a 1.2-mm-diameter drill. Cylindrical implants $1.2 \mathrm{~mm}$ in diameter and $4.0 \mathrm{~mm}$ long were prepared from pure titanium (CLINE Co., Ltd., Tokyo, Japan), sandblasted with $110-\mu \mathrm{m}$-diameter $\mathrm{AlO}_{2}$, cleaned with an ultrasonic device, and autoclaved. The implants were then inserted into the hole to a depth of $2.5 \mathrm{~mm}$ using a drill at a speed of $500 \mathrm{rpm}$, with saline irrigation to avoid heating the bone. The remaining $1.5 \mathrm{~mm}$ of the implant was covered by muscle rather than bone, and used for the connection to the load cell to measure pull-out strength. The incision wound was sutured after completion of the surgery.

Seven days before the rats were euthanized, $20 \mathrm{mg} / \mathrm{kg}$ of calcein was injected intraperitoneally to visualize new bone formation using fluorescent labeling. Nine rats in each group were randomly selected and euthanized at 2 weeks after implantation. The remaining nine rats in each group continued their diets, and were euthanized in the same 
manner at 4 weeks after implantation. All rats were subjected to pull-out testing [15, 20-22], BMD analysis, and fluorescence microscopy observation.

\subsection{Outcome measurements}

2.3.1. Pull-out strength-The pull-out strength test is described in Fig. 1. Specimens were mechanically anchored to a baseplate with self-curing resin. The setup was adjusted using a level to align the test area with the load cell such that the direction of the exerted force was as perpendicular as possible, to minimize shear forces. A 110-mm-long stainless steel wire was threaded through the hole at the top of the implant and connected to the load cell, with $50 \mathrm{~mm}$ separating the top of the implant and load cell. An Instron universal testing machine (TG-5k, Minebea Co., Kanagawa, Japan) was used for the pull-out test with a 1.0 $\mathrm{mm} / \mathrm{min}$ cross-head speed. Pull-out strength was determined as the peak force applied to detach the implant from the bone.

2.3.2. BMD and BMD color imaging-After the pull-out test, the femur was subjected to microcomputed tomography (mCT) scanning performed with an R_mCT2 device (Rigaku, Tokyo, Japan) using a $90-\mathrm{kV}$ anode electrical current at a $30-\mu \mathrm{m}$ resolution. The isotropic voxel resolution was $30 \mu \mathrm{m} \times 30 \mu \mathrm{m} \times 30 \mu \mathrm{m}$. To verify new bone formation around the implant, a $1.5-\mathrm{mm}^{2}$ area surrounding the bone where the $1.2-\mathrm{mm}$ implant was placed was scanned from a depth of $0.5-1.0 \mathrm{~mm}$ from the inner cortical bone. Thus, a cuboid of peri-implant bone with a $1.5-\mathrm{mm} \times 1.5-\mathrm{mm}$ base and a $0.5-\mathrm{mm}$ height (Fig. 2) was used to analyze BMD. R_mCT Image Analysis software (Rigaku, Tokyo, Japan) was used to generate three-dimensional models using the scanned data. A TRI/3D-BON image analyzer (Ratoc System Engineering, Tokyo, Japan) was used to calculate the BMD of the periimplant bone cuboid and generate color images depicting BMD intensity, with blue and light blue, green and yellow, and orange and red representing low, medium, and high BMD values, respectively.

2.3.3. Fluorescence microscopy observation-The femur was cut at the midpoint of the long axis using a diamond disk (Isomet; Buehler Ltd., Lake Bluff, IL, USA). The bone tissue was dehydrated in a 70\%-80\% ethanol series followed by $100 \%$ acetone, embedded in methyl methacrylate acrylic resin (Osteoresin Embedding Kit, Wako Pure Chemical Industries, Tokyo, Japan), and cured. Embedded specimens were cut into $30-\mu \mathrm{m}$ sections perpendicular to the long axis of the implant using a diamond disk, and unstained, nondemineralized specimens from the diaphyseal region were obtained. New bone formation around the implant was observed with a fluorescence microscope (BX51; Olympus, Tokyo, Japan).

2.3.4. Body weight-All 36 rats were weighed at 7, 9, and 11 weeks; the 18 surviving rats were also weighed at 13 weeks to confirm growth and good physical condition. Rats were also weighed before euthanasia.

\subsection{Statistical analysis}

Between- and within-group differences in BMD and pull-out strength at 2 and 4 weeks after implantation were analyzed by the Mann-Whitney U test. Changes in body weight were 
separately analyzed by the Friedman test to verify whether weight significantly changed over time in each group.

All statistical analyses were performed using the statistical package PASW Statistics (Version 18.0, SPSS, Chicago, IL, USA). $P$ values $<0.05$ were considered statistically significant.

\section{Results}

\subsection{Pull-out strength}

Results of the pull-out strength test are given in Fig. 3a. The pull-out strength of the Diet with SBM group at 2 weeks after implantation was $10.6 \pm 4.86 \mathrm{~N}$, approximately 1.6 times greater than that of the Diet without SBM group $(6.70 \pm 0.72 \mathrm{~N}$; between-group comparison, $P<0.05)$. The pull-out strength of the Diet with SBM group at 4 weeks after implantation was $34.1 \pm 8.85 \mathrm{~N}$, also approximately 1.6 times greater than that of the Diet without SBM group ( $23.0 \pm 4.18 \mathrm{~N}$; between-group comparison, $P<0.05)$. Both diet groups showed statistically significant increases in pull-out strength 2 to 4 weeks after implantation (withingroup comparison, $P<0.05)$.

\subsection{Bone mineral density}

BMD results are given in Fig. 3b. The BMD of the Diet with SBM group at 2 weeks after implantation was $474.13 \pm 59.39 \mathrm{mg} / \mathrm{cm}^{3}$, approximately 1.6 times greater than that of the Diet with SBM group $\left(292.04 \pm 15.60 \mathrm{mg} / \mathrm{cm}^{3}\right.$; between-group comparison, $\left.P<0.05\right)$. The BMD of the Diet With SBM group at 4 weeks after implantation was $709.36 \pm 56.65 \mathrm{mg} /$ $\mathrm{cm}^{3}$, approximately 1.5 times greater than that of the Diet with SBM group (484.64 \pm 75.59 $\mathrm{mg} / \mathrm{cm}^{3}$; between-group comparison, $P<0.05$ ). Both diet groups showed statistically significant increases in BMD from 2 to 4 weeks after implantation (within-group comparison, $P<0.05)$.

\subsection{BMD color imaging}

The BMD color image from the Diet without SBM group was primarily blue and green at 2 and 4 weeks after implantation (Figs. 4A-a and c), but was primarily yellow, with some red and blue, in the Diet with SBM group at the same time points (Figs. 4A-b and d). Given that blue/light blue, green/yellow, and orange/red represent low, medium, and high BMD, respectively, these results indicate that the BMD of peri-implant bone was higher in the Diet with SBM group than in the Diet without SBM group.

\subsection{Fluorescence microscopy observation}

Fluorescence microscopy imaging of the Diet with SBM group demonstrated more green fluorescence (indicating bone formation) than that of the Diet without SBM group at both 2 and 4 weeks after implantation (Fig. 4). As the rats aged from 2-4 weeks (Figs. 4B-a and b, and $4 \mathrm{~A}-\mathrm{a}$ and $\mathrm{b}$, respectively), the green fluorescence became more pronounced in both diet groups. 


\subsection{Body weight}

Body weight comparisons are given in Fig. 5. The body weights of both diet groups increased significantly over the feeding period (Fig. 5; Friedman test, $P<0.0001$ ). The body weights of the Diet with SBM and Diet without SBM groups were $167.18 \pm 6.58 \mathrm{~g}$ and $184.39 \pm 11.27 \mathrm{~g}$, respectively, at 2 weeks after implantation; and $193.87 \pm 13.04 \mathrm{~g}$ and $196.16 \pm 10.17 \mathrm{~g}$, respectively, at 4 weeks after implantation. The Mann-Whitney U test indicated no significant body weight difference between the groups at 2 or 4 weeks after implantation $(P>0.05)$, but also revealed significant within-group increases in body weight from 2 to 4 weeks in both groups $(P<0.05)$.

\section{Discussion}

This animal study revealed that in a rat osteoporosis model, dietary supplementation with SBM resulted in greater peri-implant BMD and pull-out strength compared with a normal diet, suggesting that SBM could contribute to bone formation around implants. These findings highlight the potential of oral supplements to accelerate bone formation after dental implant surgery in patients with osteoporosis.

Osteoporotic rats fed with SBM supplementation had 1.6 times greater pull-out strength at 2 weeks and 4 weeks after implant than those without SBM supplementation. Because the presence of SBM in the feed was the only difference in intervention between the groups, the observed difference in pull-out strength might be derived from the promotion of bone formation by SBM. Given that the body weights of both groups significantly and steadily increased during the feeding period, the rats seemed to grow normally. Some increased bone formation may be explained by the growth of the rats; however, similar growth curves were observed in both groups, suggesting that SBM worked mainly by reinforcing the bone, not by increasing body weight.

To investigate influences on pull-out strength in greater depth, BMD, BMD color imaging, and fluorescence microscopy observations were analyzed. BMD and pull-out strength in the Diet with SBM group were about 1.5-1.6 times those in the Diet without SBM group at both 2 weeks and 4 weeks. As the degree of increase in pull-out strength and BMD were similar, the increase in BMD may have resulted in the increased pull-out strength. The SBMcontaining diet had 7 times more $\mathrm{Mg}$ and 12 times more $\mathrm{Zn}$ than the Diet without SBM, and also included F, which was absent from the control diet. $\mathrm{Mg}$, Zn, and F play important roles in bone formation and resorption [23-26]; Mg deficiency results in bone loss [27]; $\mathrm{Zn}$ has been shown to reduce cathepsin and carbonic anhydrase mRNA expression, inhibiting osteoclast development [24]; and $\mathrm{F}$ has been shown to promote osteoblast differentiation by increasing total collagen content and alkaline phosphatase activity [25,26]. Mijares et al. stated that the mechanism of the SBM effect may be due to the individual and combined effects of $\mathrm{Mg}, \mathrm{Zn}$, and $\mathrm{F}$ on bone cell activities such as bone formation and resorption when released from SBM or when incorporated into newly formed bone [19]. These reports might explain why SBM-fed rats demonstrated greater BMD than fed a normal diet.

BMD color images also indicated that new bone growth around the implants was greater in the Diet with SBM group than in the Diet without SBM group. Color imaging (Figs. 4A-b 
and d) visually suggested that the implants were surrounded and fixed more quickly in the Diet with SBM group, likely resulting in the observed rapid increase in pull-out strength. This finding is consistent with the results of the fluorescence microscopy imaging (Fig. 4B), which showed the dynamics of bone remolding via the administration and subsequent incorporation of calcein fluorescent dye. Taken together, these results persuasively demonstrate that pull-out strength was greater in the Diet with SBM group than in the Diet without SBM group.

In the future, the number of implant treatments performed in older patients with osteoporosis is expected to increase, reflecting an aging society. SBM was originally developed for the prevention and treatment of osteoporosis. Therefore, from the perspective of both osteoporosis and implant treatment, the SBM supplement appears to be a promising subject for further study.

\section{Conclusion}

Within its limitations, this study concludes that the dietary supplement SBM, developed for osteoporosis, is effective in accelerating peri-implant bone formation in a rat model of osteoporosis.

\section{Acknowledgments}

This work was supported in part by KAKENHI (15H06645) from the Japan Society for the Promotion of Science and NIAMS/NIH Research grant no. 2R01 AR056208 (Y. Zhang). The authors gratefully thank Prof. Toshiro Sakae for discussions of this article.

\section{References}

1. Orimo H, Nakamura T, Hosoi T, Iki M, Uenishi K, Endo N, et al. Japanese 2011 guidelines for prevention and treatment of osteoporosis--executive summary. Archives of osteoporosis. 2012; 7:320. [PubMed: 23203733]

2. Kim IK. Health innovation for aging society. Healthcare informatics research. 2015; 21:63-6. [PubMed: 25995957]

3. Takamoto A, Tokikazu T, Dainobu K, Takechi M, Ohta K, Ninimiya Y, et al. Questionnaire Survery about Uneasiness and Satisfaction to Bone Augmentation Surgery for Oral Implant. J Hiroshima Univ Dent Soc. 2012; 44:29-35.

4. Johansson C, Albrektsson T. Integration of screw implants in the rabbit: a 1-year follow-up of removal torque of titanium implants. The International journal of oral \& maxillofacial implants. 1987; 2:69-75. [PubMed: 3481352]

5. Raisz LG. Pathogenesis of osteoporosis: concepts, conflicts, and prospects. The Journal of clinical investigation. 2005; 115:3318-25. [PubMed: 16322775]

6. Fini M, Giavaresi G, Torricelli P, Borsari V, Giardino R, Nicolini A, et al. Osteoporosis and biomaterial osteointegration. Biomedicine $\&$ pharmacotherapy = Biomedecine $\&$ pharmacotherapie. 2004; 58:487-93. [PubMed: 15511604]

7. Blomqvist JE, PA, Isaksson S, Linde A, Hansson B-G. Factors in implant integration failure after bone grafting. Int J Oral Maxillofac Surg. 1996; 25:63-8. [PubMed: 8833304]

8. Roccuzzo M, Aglietta M, Cordaro L. Implant loading protocols for partially edentulous maxillary posterior sites. The International journal of oral \& maxillofacial implants. 2009; 24(Suppl):147-57. [PubMed: 19885442] 
9. Cordaro L, Torsello F, Roccuzzo M. Implant loading protocols for the partially edentulous posterior mandible. The International journal of oral \& maxillofacial implants. 2009; 24(Suppl):158-68. [PubMed: 19885443]

10. Shibata Y, Tanimoto Y. A review of improved fixation methods for dental implants. Part I: Surface optimization for rapid osseointegration. Journal of prosthodontic research. 2015; 59:20-33. [PubMed: 25530606]

11. Shibata Y, Tanimoto Y, Maruyama N, Nagakura M. A review of improved fixation methods for dental implants. Part II: biomechanical integrity at bone-implant interface. Journal of prosthodontic research. 2015; 59:84-95. [PubMed: 25797023]

12. Ogawa T. Ultraviolet photofunctionalization of titanium implants. The International journal of oral \& maxillofacial implants. 2014; 29:e95-102. [PubMed: 24451893]

13. Suzuki S, Kobayashi H, Ogawa T. Implant stability change and osseointegration speed of immediately loaded photofunctionalized implants. Implant dentistry. 2013; 22:481-90. [PubMed: 24021973]

14. Ogawa T, Zhang X, Naert I, Vermaelen P, Deroose CM, Sasaki K, et al. The effect of whole-body vibration on peri-implant bone healing in rats. Clinical oral implants research. 2011; 22:302-7. [PubMed: 21044169]

15. Watanabe T, Nakada H, Takahashi T, Fujita K, Tanimoto Y, Sakae T, et al. Potential for acceleration of bone formation after implant surgery by using a dietary supplement: an animal study. Journal of oral rehabilitation. 2015; 42:447-53. [PubMed: 25572652]

16. Ayukawa Y, Ogino Y, Moriyama Y, Atsuta I, Jinno Y, Kihara M, et al. Simvastatin enhances bone formation around titanium implants in rat tibiae. Journal of oral rehabilitation. 2010; 37:123-30. [PubMed: 19889034]

17. Takehiro Watanabe HN, Takahashi Takahiro, Fujita Kanami, Tanimoto Yasuhiro, Toshirou, Sakae SK, Kawai Yasuhiko. The Influence of Synthetic Bone Mineral to Bone Formation. Int J Oral-Med Sci. 2015; 13:89-93.

18. LeGeros RZ. Patent. 2008: US 7, B2 (submitted 2004,, issued September 2 UAC, March, 12 Cp$\mathrm{bb}$, incorporating magnesium $\mathrm{z}$, fluoride and carbonate. ed., p. of.

19. Mijares D, Kulkarni A, Lewis K, Yao F, Xi Q, Tannous S, et al. Oral bone loss induced by mineral deficiency in a rat model: effect of a synthetic bone mineral (SBM) preparation. Archives of oral biology. 2012; 57:1264-73. [PubMed: 22424637]

20. Alghamdi HS, Cuijpers VM, Wolke JG, van den Beucken JJ, Jansen JA. Calcium-phosphate-coated oral implants promote osseointegration in osteoporosis. Journal of dental research. 2013; 92:9828. [PubMed: 24056224]

21. Zacchetti G, Wiskott A, Cugnoni J, Botsis J, Ammann P. External mechanical microstimuli modulate the osseointegration of titanium implants in rat tibiae. BioMed research international. 2013; 2013:234093. [PubMed: 24369009]

22. Aparicio C, Padros A, Gil FJ. In vivo evaluation of micro-rough and bioactive titanium dental implants using histometry and pull-out tests. Journal of the mechanical behavior of biomedical materials. 2011; 4:1672-82. [PubMed: 22098868]

23. Rude RK, Gruber HE. Magnesium deficiency and osteoporosis: animal and human observations. The Journal of nutritional biochemistry. 2004; 15:710-6. [PubMed: 15607643]

24. Yamada Y, Ito A, Kojima H, Sakane M, Miyakawa S, Uemura T, et al. Inhibitory effect of Zn2+ in zinc-containing beta-tricalcium phosphate on resorbing activity of mature osteoclasts. Journal of biomedical materials research Part A. 2008; 84:344-52. [PubMed: 17618520]

25. Inoue M, LeGeros RZ, Inoue M, Tsujigiwa H, Nagatsuka H, Yamamoto T, et al. In vitro response of osteoblast-like and odontoblast-like cells to unsubstituted and substituted apatites. Journal of biomedical materials research Part A. 2004; 70:585-93. [PubMed: 15307163]

26. Miyagi M, Tsuruda K, Kawamura M, Morishita M, Iwamoto Y. Effects of fluoride intake on the mineral content, acid solubility and resorption caused by experimental periodontitis of rat alveolar bone. Archives of oral biology. 1994; 39:163-6. [PubMed: 8185502]

27. Rude RK, Gruber HE, Norton HJ, Wei LY, Frausto A, Kilburn J. Dietary magnesium reduction to $25 \%$ of nutrient requirement disrupts bone and mineral metabolism in the rat. Bone. 2005; 37:2119. [PubMed: 15923157] 


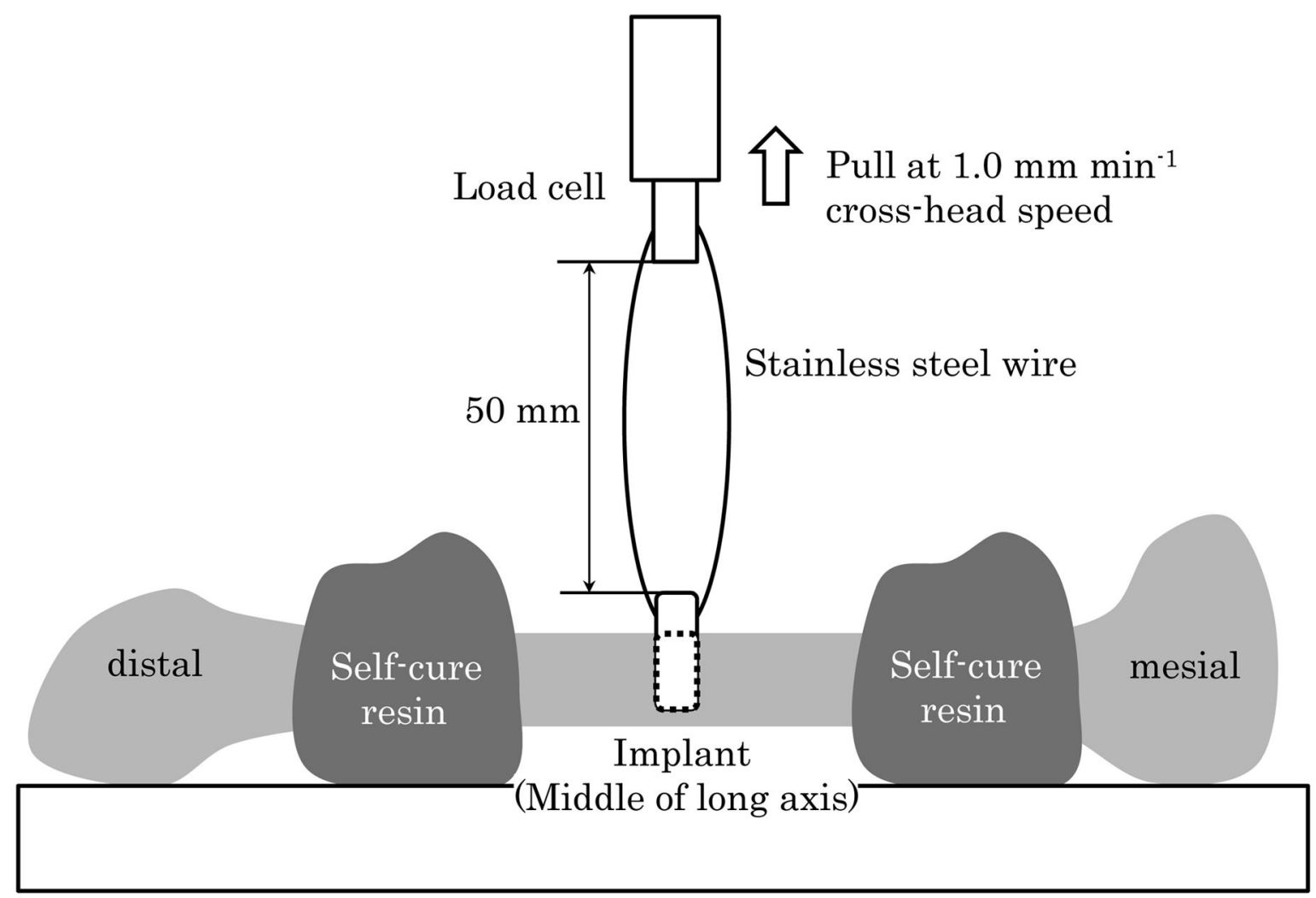

Fig. 1.

The pull-out strength test. Specimens are mechanically anchored to a baseplate with selfcuring resin. The load cell is positioned to pull the implant as perpendicularly as possible to minimize shear forces, at a cross-head speed of $1.0 \mathrm{~mm} / \mathrm{min}^{-1}$. 


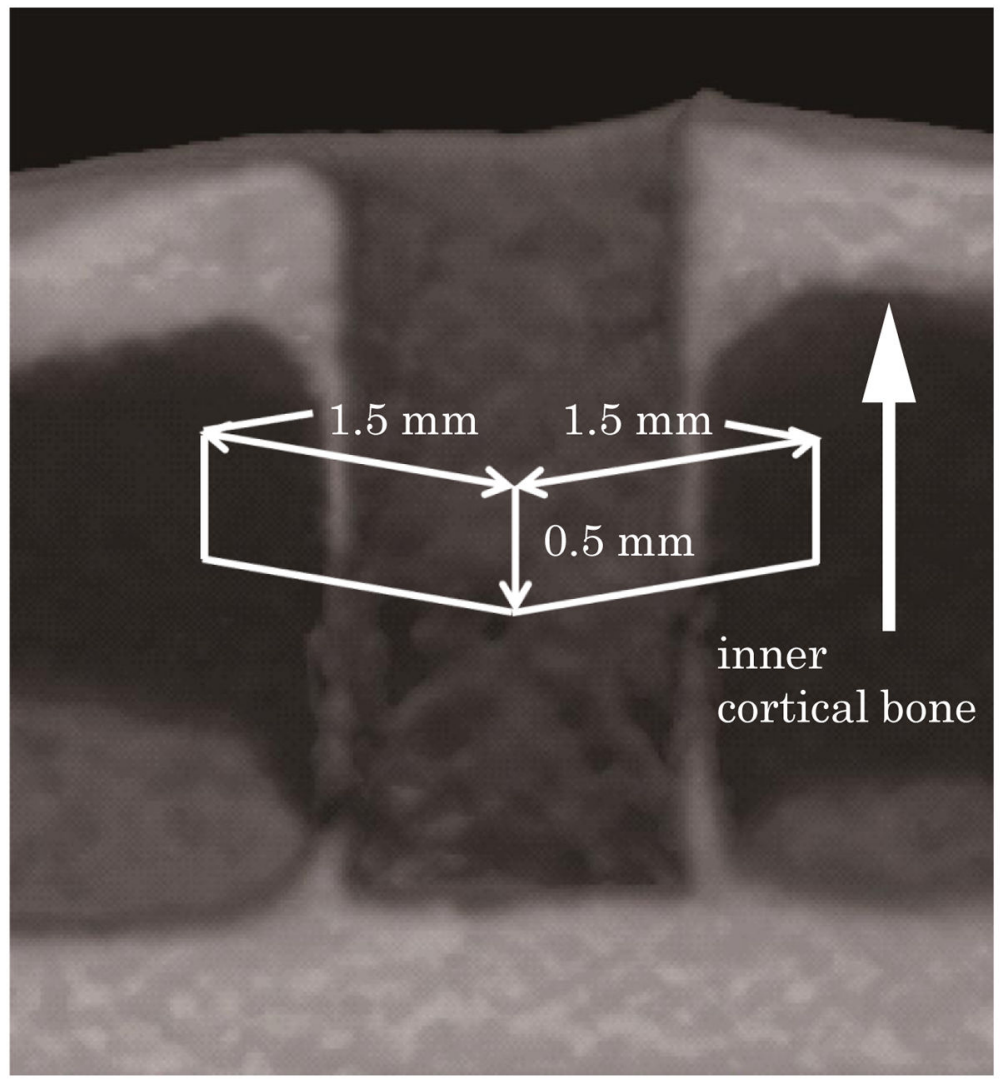

Fig. 2. Peri-implant bone scanning for BMD analyses

A $1.5-\mathrm{mm}^{2}$ area surrounding the bone socket where a $1.2-\mathrm{mm}$ implant had been placed was three-dimensionally scanned from a depth of $0.5-1.0 \mathrm{~mm}$ from the inner cortical bone. Thus, a cuboid of peri-implant bone with a $1.5 \mathrm{~mm} \times 1.5 \mathrm{~mm}$ base and a 0.5 -mm height was scanned. BMD, bone mineral density. 
(a)

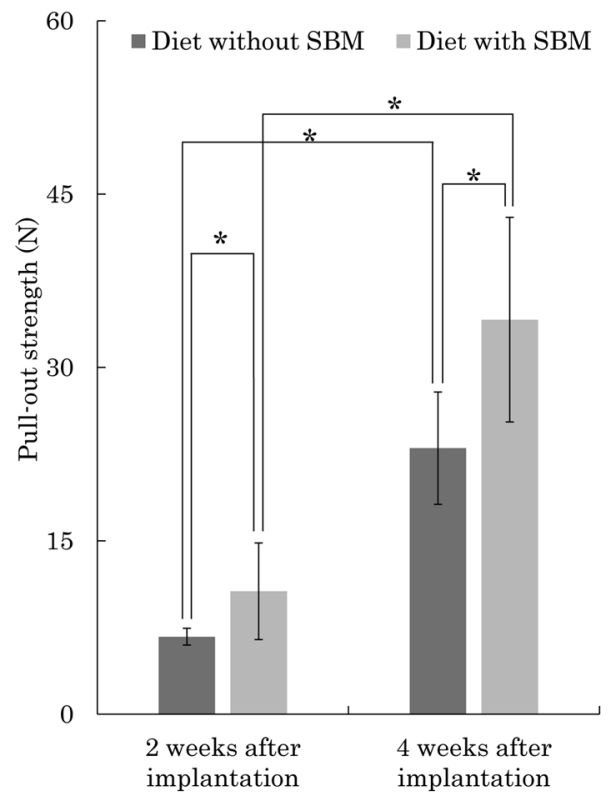

(b)

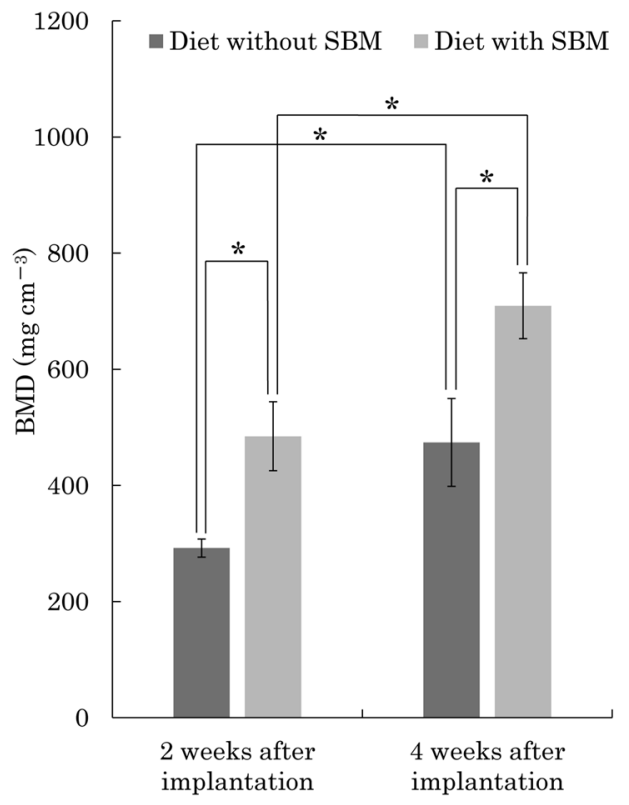

Fig. 3. Between- and within-group comparisons of BMD and pull-out strength Results of the pull-out strength test (a): Pull-out strength in the Diet with SBM group at 4 weeks after implantation is approximately 1.6 times that in the Diet without SBM group (between-group comparisons, $P<0.05$ ). Both diet groups show a statistically significant increase in pull-out strength from 2 to 4 weeks after implantation (within-group comparison, $P<0.05)$.

BMD Results (b): BMD in the Diet with SBM group at 2 weeks after implantation is approximately 1.6 times that in the Diet without SBM group (between-group comparison, $P$ $<0.05)$. BMD in the Diet with SBM group at 4 weeks after implantation is approximately 1.5 times greater than that in the Diet without SBM group (between-group comparisons, $P<$ 0.05). Both diet groups show statistically significant increases in BMD from 2 to 4 weeks after implantation (within-group comparison, $P<0.05$ ).

BMD, bone mineral density; SBM, synthetic bone mineral. 


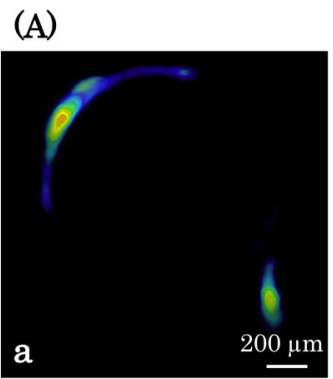

Diet without SBM

2 weeks after implantation

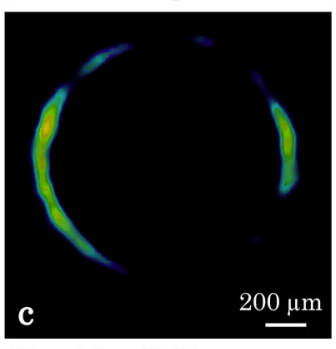

Diet without SBM

4 weeks after implantation

(B)

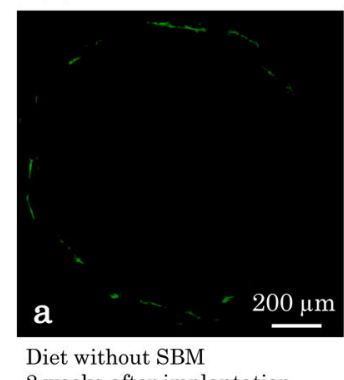

2 weeks after implantation

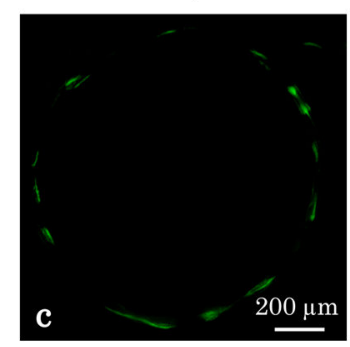

Diet without SBM

4 weeks after implantation

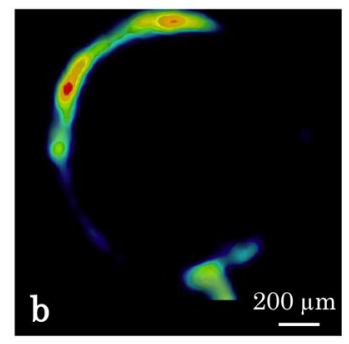

Diet with SBM

2 weeks after implantation

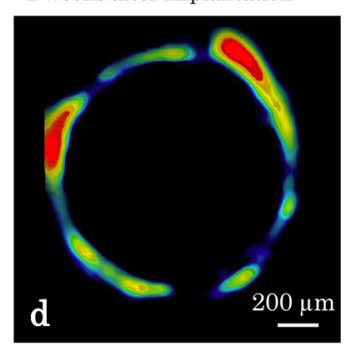

Diet with SBM

4 weeks after implantation

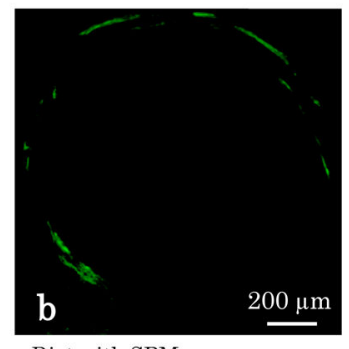

Diet with SBM

2 weeks after implantation

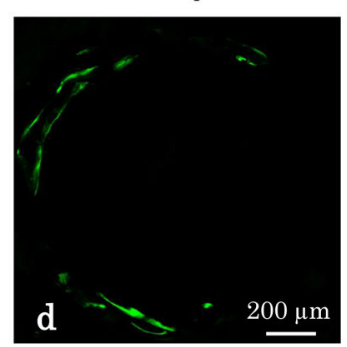

Diet with SBM

4 weeks after implantation

Fig. 4. BMD color imaging and fluorescent imaging

(A), BMD color image observations. The Diet without SBM group color image is predominantly blue and green at 2 and 4 weeks after implantation (Figs. 4A-a and c);

however, the Diet with SBM group color image is predominantly yellow, with some red and blue, at 2 and 4 weeks after implantation (Figs. 4A-b and d). Blue/light blue, green/yellow, and orange/red represent low, medium, and high BMD values, respectively.

Fluorescence images. (B): Fluorescence microscopy imaging of the Diet with SBM group has more green fluorescence (indicating new bone formation) than that of the Diet without SBM group at both 2 and 4 weeks after implantation (Fig. 4). As the rats age from 2 to 4 
weeks (Figs. 4B-a and b, and 4A-a and b, respectively), green fluorescence becomes more intense in both diet groups.

BMD, bone mineral density; SBM, synthetic bone mineral. 


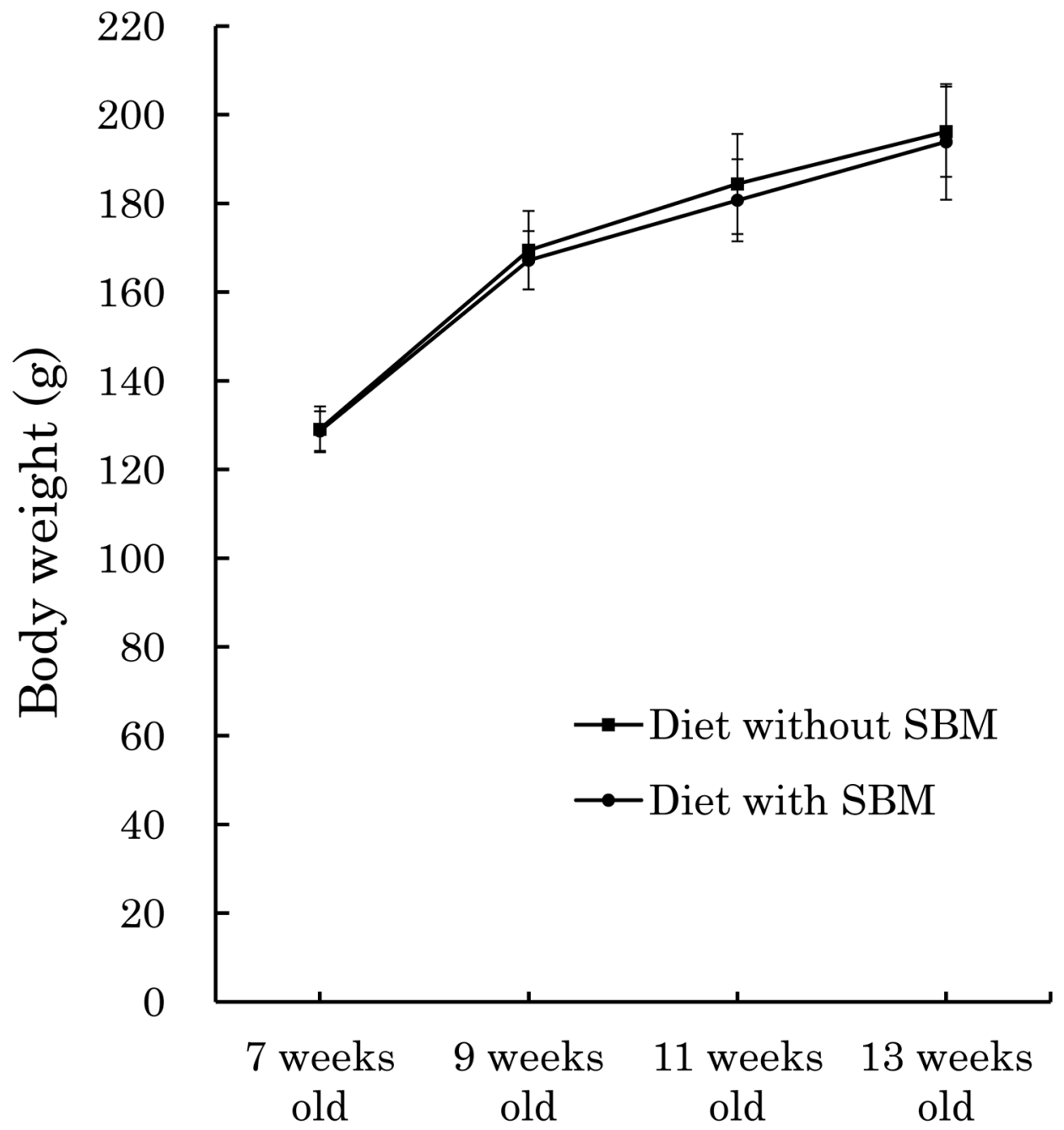

Fig. 5.

Body weight changes in the rats. Body weight significantly increases in both the Diet with SBM and Diet without SBM groups over the feeding period $(P<0.05)$. Significant body weight differences are not observed between the groups at 2 and 4 weeks after implantation. SBM, synthetic bone mineral. 

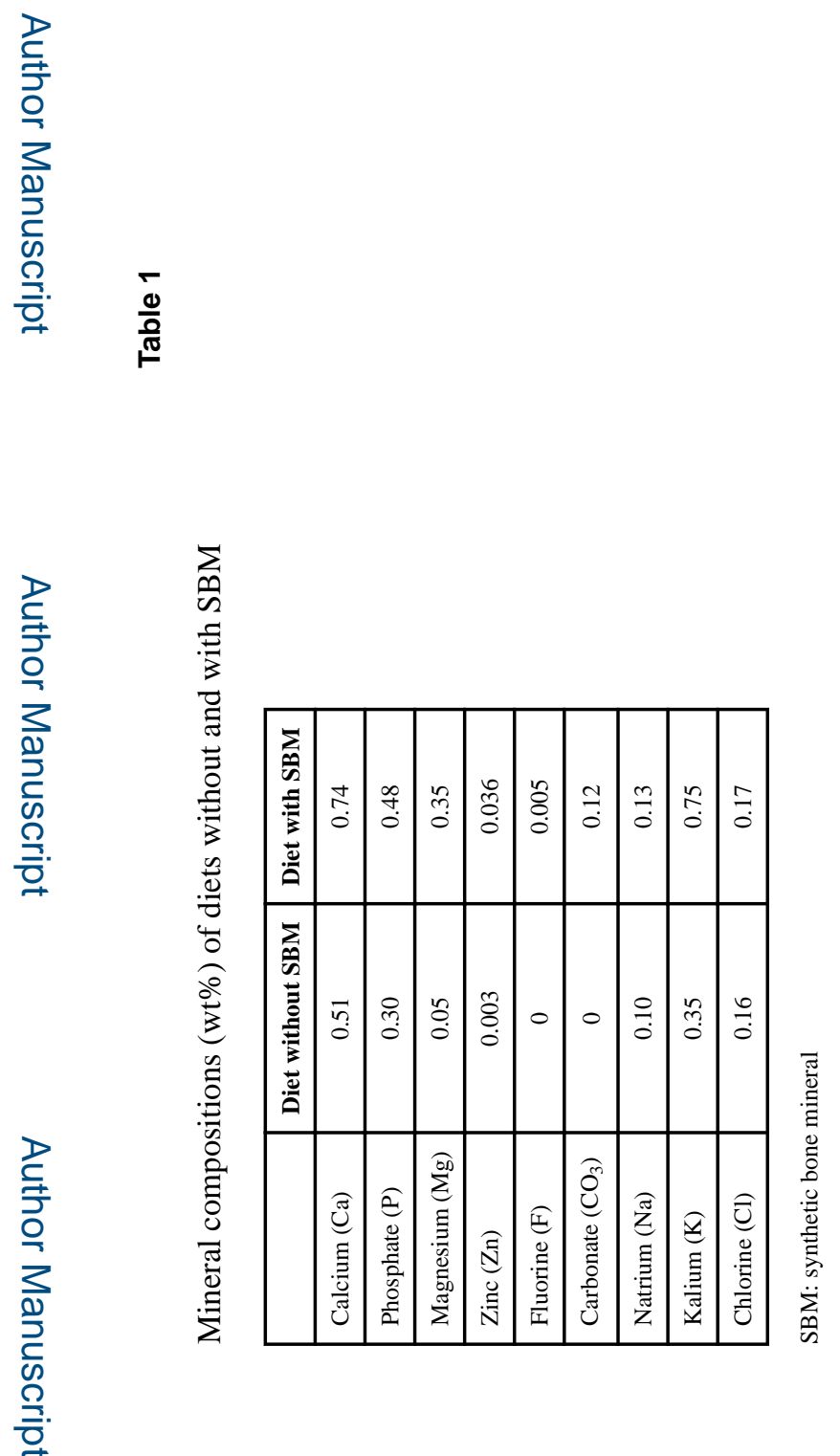

J Prosthodont Res. Author manuscript; available in PMC 2017 April 01. 